\title{
Pulsed Electron Beams for Mitigating Damage in Next-generation Electronic Materials
}

\author{
Elisah VandenBussche, Catherine Clark, Russell Holmes and David Flannigan
}

University of Minnesota, Minneapolis, Minnesota, United States

A largely unexplored area pertinent to mitigating electron-beam damage involves leveraging new TEM methods that can be used to take advantage of strongly temporally-dependent processes. One such method which has been recently demonstrated is the use of temporally-modulated electron beams, delivering electron doses with prescribed regularity in place of a thermionic beam in which electron emission is randomly distributed in time. Such beams have been used to illuminate polymer crystals for unusually prolonged exposures with apparent minimal damage [1]. In addition, for the prototypical Ziegler-Natta catalyst support $\mathrm{MgCl}_{2}$, delivering dose via well-timed, few-electron packets separated in time by 160 ps was shown to aid in reduction of accumulated phonon thermal energy, thereby suppressing a commonlyencountered damage-induced phase change [2]. In this way, an increase in the critical total dose by nearly two orders of magnitude was observed. It has also been demonstrated that delivery of timed single-electron packets separated in time by up to $100 \mu$ s leads to a reduction in structural damage to model paraffin crystals $\left(\mathrm{C}_{36} \mathrm{H}_{74}\right)$ by nearly a factor of two as compared to conventional thermionic (random) emission for the same total dose [3]. The same improvement was observed when the time between packets was reduced to $5 \mu \mathrm{s}$, and threshold levels for beneficial effects with respect to electron-packet number density were identified. Little to no specimen damage has also been observed in pulsed $\mathrm{MeV}$ electron diffraction experiments conducted at extremely low repetition rates [4]. In addition to reducing damage for a given total dose, pulsed beams can potentially be used to access new information about damage mechanisms. For example, one method for producing pulsed electron beams in the TEM is by training a femtosecond pulsed laser on the source material in the gun region (Figure 1a). Importantly, the range of pulsed-electron beam parameters that can be covered in this way is directly dependent upon the pulsed-laser properties; time between electron packets is directly tied to laser repetition rate, and fine control over the number of electrons per packet is controlled with laser fluence. This in turn amounts to control over both the specimen relaxation time between packets and the instantaneous dose rate, respectively. Indeed, this enables exploration of factors such as specimen heating and cumulative, spatially-common energy-deposition effects, as initially explored in the model paraffin systems [3].

In addition to discussing the use of pulsed beams to mediate and elucidate damage in molecular materials, such as highly-sensitive saturated hydrocarbons, we explore extension of this technique to next-generation electronic materials. A class of such materials for which the methods described above are particularly well-suited are hybrid organic-inorganic perovskites (HOIPs), which have achieved lab-scale photovoltaic power conversion efficiencies of over $25 \%$ using low-temperature solution-based syntheses [5]. However, the organic components and van der Waals bonding critical to the hybrid nature of the material renders HOIPs susceptible to degradation under various environmental conditions, including electron-beam irradiation [6]. As shown in Figure 1b, diffraction-peak intensity can be tracked over time in order to assess damage, enabling quantitative comparison between thermionic and pulsed beams and between pulsed beams of various temporal profiles. Temporal modulation methods which can reduce damage are indeed pertinent to improving high-resolution characterization of sensitive perovskite materials, but more importantly, degradation mechanisms are not fully understood due to convolution of multiple processes occurring simultaneously in both space and time. For example, volatilization and migration of iodide ions results in a superlattice with halide vacancies, the formation of which is highly dose-rate dependent [7]. 
This intermediate phase subsequently decomposes further through loss of additional halide ions and organic cations, ultimately transforming into pure metal halide. The timescales associated with this are not yet known, but the dose-rate dependence even at dose rates as low as $0.5 \mathrm{e} \cdot \AA^{-2} \cdot \mathrm{s}^{-1}$ suggests that energy deposition, for example by multiple electrons arriving rapidly relative to the timescale of energy dissipation, can lead to enhancement of the rate of damage. As perovskites have been shown to degrade more rapidly under thermal heating and have relatively low thermal conductivities, this energy deposition via electron dosing may contribute to thermally-induced damage in the perovskites as well $[8,9]$. Instability under light, electrical bias, and heat are limiting factors in implementation of HOIPs as widespread photovoltaic materials, and electron-beam irradiation is ideally suited to study these degradation mechanisms because it enables ions to overcome binding and diffusion barriers, thus degrading the structure. In this way, insight into electron-beam degradation mechanisms is of both fundamental and practical importance for understanding physical processes in perovskites and enabling their widespread implementation [10].
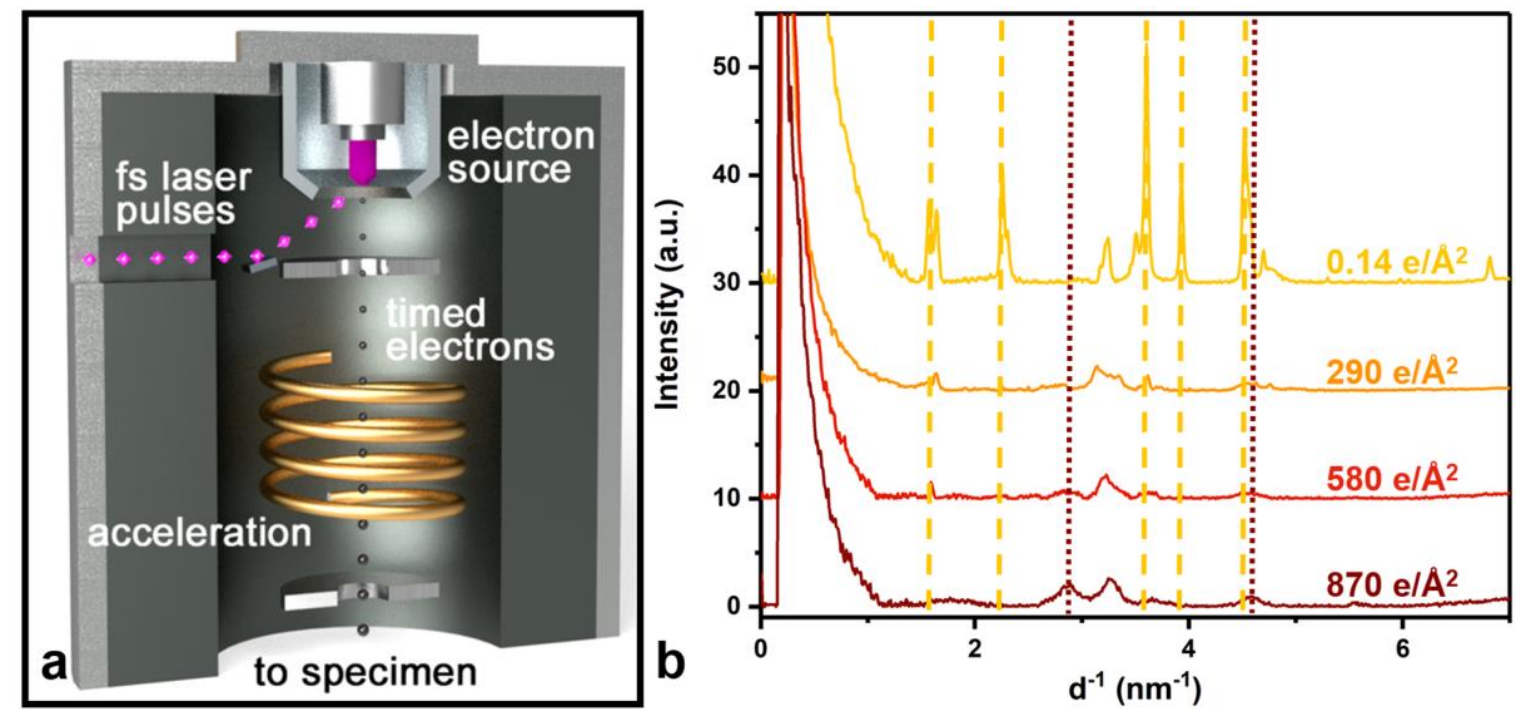

Figure 1. Pulsed beams and radiation damage in advanced materials. (a) Schematic of the source region of the pulsed-beam TEM. The fs laser pulses enter through an optical port and are directed onto the source to generate timed electron packets with narrow number-density distributions. The packets are then accelerated and sent to the specimen using conventional methods. (b) Progressive azimuthally integrated diffraction patterns for a $\mathrm{CH} 3 \mathrm{NH} 3 \mathrm{PbI} 3$ specimen being irradiated at a dose rate of 0.14 e. $\AA$ - $2 \cdot \mathrm{s}-$ 1. Representative diffraction patterns are shown after 0.14 e $\cdot \AA-2$ (yellow), 290 e $\cdot \AA-2$ (orange), 580 e $\cdot \AA$ 2 (bright red), and 870 e. $\AA-2$ (dark red) of total dose. Vertical yellow dashed lines denote peaks which are characteristic of the $\mathrm{CH} 3 \mathrm{NH} 3 \mathrm{PbI} 3$ specimen, which decay as accumulated dose increases. Peaks denoted correspond to the $001,011,012,112$, and 022 reflections in order from left to right. Vertical maroon dotted lines denote peaks which are characteristic of the PbI2 degradation product phase, which increase in intensity as accumulated dose increases. Peaks denoted correspond to the 011 and 111 reflections from left to right.

\section{References}

[1] O.-H. Kwon, et al., Proc. Natl. Acad. Sci. U.S.A. 108 (2011), 6026.

[2] C. Kisielowski, et al., Adv. Funct. Mater. 29 (2019), 1807818.

[3] E. J. VandenBussche and D. J. Flannigan, Nano Lett. 19 (2019), 6687.

[4] X. Yang, et al., Sci. Rep. 9 (2019), 17223. 
[5] Best Research-Cell Efficiency Chart, https://www.nrel.gov/pv/cell-efficiency.html (accessed February 17, 2020).

[6] J. Ran, et al., Adv. Energy Mater. (2020), 1903191. (Early View)

[7] S. Chen, et al., Nat. Commun. 9 (2018), 4807.

[8] A. Pisoni, et al., J. Phys. Chem. Lett. 5 (2014), 2488.

[9] G. Divitini, et al., Nat. Energy 1 (2016), 15012.

[10] This material is based on work supported by the U.S. Department of Energy, Office of Science, Office of Basic Energy Sciences under Award No. DE-SC0018204. This material is based upon work supported by the National Science Foundation Graduate Research Fellowship Program under Grant No. DGE1348264 . 